\title{
A New Mathematical Model and its Solvability Test on Computer
}

\author{
Sheng Zhang ${ }^{1,}$, , Chi Tian ${ }^{1, \text { b }}$ \\ ${ }^{1}$ School of Mathematics and Physics, Bohai University, Jinzhou, 121013, China \\ aemail: szhangchina@126.com, bemail: 6426357@163.com
}

Keywords: Solvability; Mathematical Model; Mathematica 4.0

\begin{abstract}
It is interesting to test the solvability of nonlinear differential equations. In this paper, a new mathematical model passes through the test of solvability on computer. To test the solvability of the mathematical model, Mathematica 4.0 software system combined with a subsidiary equation method is utilized. At the same time, some solutions are obtained including hyperbolic function solutions, trigonometric function solutions and rational solution. This paper shows that the Mathematica 4.0 software system combined with the subsidiary equation method can provide a preferred mathematical tool for testing the solvability of some new nonlinear differential equations.
\end{abstract}

\section{Introduction}

In 2012, Shan and Zhu [1] derived a new mathematical model:

$$
\begin{aligned}
u_{t}= & 18 v_{x x x}-36(v u)_{x}-u_{7 x}+49 u_{x} u_{x x x x}+14 u u_{5 x}+84 u_{x x} u_{x x x}-70 u_{x}^{3} \\
& -252 u u_{x} u_{x x}-56 u^{2} u_{x x x}+\frac{224}{3} u^{3} u_{x}, \\
v_{t}= & -36 v v_{x}+v_{x} v_{x x x x}+3 v u_{5 x}-12 v_{x} u u_{x x}-72 v u_{x} u_{x x}-36 v u u_{x x x}-6 v_{x} u_{x}^{2}+\frac{32}{3} u^{3} v_{x}+96 v u^{2} u_{x} .
\end{aligned}
$$

To the best of our knowledge, Eqs. (1) and (2) have not been solved. It is well known that many physical phenomena can be usually described by nonlinear partial differential equations (PDEs). Researchers often investigate exact solutions of such nonlinear PDEs to gain more insight into these physical phenomena for further applications. So, solving nonlinear PDEs is interesting. Since the initial-value problem of the Korteweg-de Vries (KdV) equation was solved by Gardner, Green, Kruskal and Miura [2] in 1965, many effective methods have been proposed for nonlinear PDEs, such as Bäcklund transformation [3], Hirota's bilinear method [4], homogeneous balance method [5], tanh-function method [6], Jacobi elliptic function expansion method [7], and others [8-17]. Generally speaking, it is hard to exactly solve a nonlinear PDE because of the complexity of the PDE itself. In this paper, we shall consider a special case of Eqs. (1) and (2) when $v=0$ :

$$
u_{t}=-u_{7 x}+49 u_{x} u_{x x x x}+14 u u_{5 x}+84 u_{x x} u_{x x x}-70 u_{x}^{3}-252 u u_{x} u_{x x}-56 u^{2} u_{x x x}+\frac{224}{3} u^{3} u_{x},
$$

for testing its solvability by using Mathematica 4.0 software system combined with a subsidiary equation method. As a result, five solutions are obtained including hyperbolic function solutions, trigonometric function solutions and rational solution.

\section{Solvability and Exact Solutions}

Firstly, we take advantage of the following travelling wave transformation:

$u=u(\xi), \quad \xi=k x+\omega t$,

Eq. (3) becomes

$$
\begin{aligned}
\omega u^{\prime}= & -k^{7} u^{(7)}+49 k^{5} u^{\prime} u^{(4)}+14 k^{5} u u^{(5)}+84 k^{5} u^{\prime \prime} u^{\prime \prime \prime}-70 k^{3} u^{\prime 3}-252 k^{3} u u^{\prime} u^{\prime \prime} \\
& -56 k^{3} u^{2} u^{\prime \prime \prime}+\frac{224}{3} k u^{3} u^{\prime} .
\end{aligned}
$$

Secondly, we suppose that Eq. (5) has a solution in the form: 
$u(\xi)=a_{0}+a_{1} \phi(\xi)+a_{2} \phi^{2}(\xi)+\cdots+a_{m} \phi^{m}(\xi)$,

where $a_{0}, a_{1}, a_{2}, \cdots, a_{m}$ are constants to be determined later, $m$ is an undetermined integer, while $\phi(\xi)$ satisfies the subsidiary equation

$\phi^{\prime}(\xi)=\sigma+\phi^{2}(\xi), \quad \sigma=$ constant.

Balancing the highest order linear term $u^{(7)}$ with the highest order nonlinear term $u^{3} u^{\prime}$ in Eq. (5) yields

$7+m=4 m+1$

which gives

$m=2$.

Running Mathematica 4.0, from Eqs. (6) and (7) we have

$$
\begin{aligned}
u^{\prime \prime} & =6 a_{2} \phi^{2} \phi^{\prime}+2 a_{1} \phi \phi^{\prime}+2 a_{2} \sigma \phi^{\prime} \\
& =6 a_{2} \sigma \phi^{2}+6 a_{2} \phi^{4}+2 a_{1} \sigma \phi+2 a_{1} \phi^{3}+2 a_{2} \sigma^{2}+2 a_{2} \sigma \phi^{2} \\
& =6 a_{2} \phi^{4}+2 a_{1} \phi^{3}+8 a_{2} \sigma \phi^{2}+2 a_{1} \sigma \phi+2 a_{2} \sigma^{2},
\end{aligned}
$$

$u^{\prime \prime \prime}=24 a_{2} \phi^{3} \phi^{\prime}+6 a_{1} \phi^{2} \phi^{\prime}+16 a_{2} \sigma \phi \phi^{\prime}+2 a_{1} \sigma \phi^{\prime}$

$$
\begin{aligned}
& =24 a_{2} \sigma \phi^{3}+24 a_{2} \phi^{5}+6 a_{1} \sigma \phi^{2}+6 a_{1} \phi^{4}+16 a_{2} \sigma^{2} \phi+16 a_{2} \sigma \phi^{3}+2 a_{1} \sigma^{2}+2 a_{1} \sigma \phi^{2} \\
& =24 a_{2} \phi^{5}+6 a_{1} \phi^{4}+40 a_{2} \sigma \phi^{3}+8 a_{1} \sigma \phi^{2}+16 a_{2} \sigma^{2} \phi+2 a_{1} \sigma^{2},
\end{aligned}
$$

$u^{(4)}=120 a_{2} \phi^{4} \phi^{\prime}+24 a_{1} \phi^{3} \phi^{\prime}+120 a_{2} \sigma \phi^{2} \phi^{\prime}+16 a_{1} \sigma \phi \phi^{\prime}+16 a_{2} \sigma^{2} \phi^{\prime}$

$=120 a_{2} \sigma \phi^{4}+120 a_{2} \phi^{6}+24 a_{1} \sigma \phi^{3}+24 a_{1} \phi^{5}+120 a_{2} \sigma^{2} \phi^{2}+120 a_{2} \sigma \phi^{4}+16 a_{1} \sigma^{2} \phi$

$+16 a_{1} \sigma \phi^{3}+16 a_{2} \sigma^{3}+16 a_{2} \sigma^{2} \phi^{2}$

$=120 a_{2} \phi^{6}+24 a_{1} \phi^{5}+240 a_{2} \sigma \phi^{4}+40 a_{1} \sigma \phi^{3}+136 a_{2} \sigma^{2} \phi^{2}+16 a_{1} \sigma^{2} \phi+16 a_{2} \sigma^{3}$,

$u^{(5)}=720 a_{2} \phi^{5} \phi^{\prime}+120 a_{1} \phi^{4} \phi^{\prime}+960 a_{2} \sigma \phi^{3} \phi^{\prime}+120 a_{1} \sigma \phi^{2} \phi^{\prime}+272 a_{2} \sigma^{2} \phi \phi^{\prime}+16 a_{1} \sigma^{2} \phi^{\prime}$

$=720 a_{2} \sigma \phi^{5}+720 a_{2} \phi^{7}+120 a_{1} \sigma \phi^{4}+120 a_{1} \phi^{6}+960 a_{2} \sigma^{2} \phi^{3}+960 a_{2} \sigma \phi^{5}$

$+120 a_{1} \sigma^{2} \phi^{2}+120 a_{1} \sigma \phi^{4}+272 a_{2} \sigma^{3} \phi+272 a_{2} \sigma^{2} \phi^{3}+16 a_{1} \sigma^{3}+16 a_{1} \sigma^{2} \phi^{2}$

$=720 a_{2} \phi^{7}+120 a_{1} \phi^{6}+1680 a_{2} \sigma \phi^{5}+240 a_{1} \sigma \phi^{4}+1232 a_{2} \sigma^{2} \phi^{3}$

$+136 a_{1} \sigma^{2} \phi^{2}+272 a_{2} \sigma^{3} \phi+16 a_{1} \sigma^{3}$,

$u^{(6)}=5040 a_{2} \phi^{6} \phi^{\prime}+720 a_{1} \phi^{5} \phi^{\prime}+8400 a_{2} \sigma \phi^{4} \phi^{\prime}+960 a_{1} \sigma \phi^{3} \phi^{\prime}+3696 a_{2} \sigma^{2} \phi^{2} \phi^{\prime}$

$+272 a_{1} \sigma^{2} \phi \phi^{\prime}+272 a_{2} \sigma^{3} \phi^{\prime}$

$=5040 a_{2} \sigma \phi^{6}+5040 a_{2} \phi^{8}+720 a_{1} \sigma \phi^{5}+720 a_{1} \phi^{7}+8400 a_{2} \sigma^{2} \phi^{4}+8400 a_{2} \sigma \phi^{6}$

$+960 a_{1} \sigma^{2} \phi^{3}+960 a_{1} \sigma \phi^{5}+3696 a_{2} \sigma^{3} \phi^{2}+3696 a_{2} \sigma^{2} \phi^{4}+272 a_{1} \sigma^{3} \phi+272 a_{1} \sigma^{2} \phi^{3}$

$+272 a_{2} \sigma^{4}+272 a_{2} \sigma^{3} \phi^{2}$

$=5040 a_{2} \phi^{8}+720 a_{1} \phi^{7}+13440 a_{2} \sigma \phi^{6}+1680 a_{1} \sigma \phi^{5}+12096 a_{2} \sigma^{2} \phi^{4}+1232 a_{1} \sigma^{2} \phi^{3}$

$+3968 a_{2} \sigma^{3} \phi^{2}+272 a_{1} \sigma^{3} \phi+272 a_{2} \sigma^{4}$,

$u^{(7)}=40320 a_{2} \phi^{7} \phi^{\prime}+5040 a_{1} \phi^{6} \phi^{\prime}+80640 a_{2} \sigma \phi^{5} \phi^{\prime}+8400 a_{1} \sigma \phi^{4} \phi^{\prime}+48384 a_{2} \sigma^{2} \phi^{3} \phi^{\prime}$

$+3696 a_{1} \sigma^{2} \phi^{2} \phi^{\prime}+7936 a_{2} \sigma^{3} \phi \phi^{\prime}+272 a_{1} \sigma^{3} \phi^{\prime}$

$=40320 a_{2} \sigma \phi^{7}+40320 a_{2} \phi^{9}+5040 a_{1} \sigma \phi^{6}+5040 a_{1} \phi^{8}+80640 a_{2} \sigma^{2} \phi^{5}+80640 a_{2} \sigma \phi^{7}$

$+5040 a_{1} \sigma \phi^{6}+8400 a_{1} \sigma^{2} \phi^{4}+8400 a_{1} \sigma \phi^{6}+48384 a_{2} \sigma^{3} \phi^{3}+48384 a_{2} \sigma^{2} \phi^{5}$ 


$$
\begin{aligned}
& +3696 a_{1} \sigma^{3} \phi^{2}+3696 a_{1} \sigma^{2} \phi^{8}+7936 a_{2} \sigma^{4} \phi+7936 a_{2} \sigma^{3} \phi^{3}+272 a_{1} \sigma^{4}+272 a_{1} \sigma^{3} \phi^{2} \\
= & 40320 a_{2} \phi^{9}+5040 a_{1} \phi^{8}+120960 a_{2} \sigma \phi^{7}+13440 a_{1} \sigma \phi^{6}+129024 a_{2} \sigma^{2} \phi^{5} \\
& +12096 a_{1} \sigma^{2} \phi^{4}+56320 a_{2} \sigma^{3} \phi^{3}+3968 a_{1} \sigma^{3} \phi^{2}+7936 a_{2} \sigma^{4} \phi+272 a_{1} \sigma^{4} .
\end{aligned}
$$

Substituting Eqs. (6), (10)-(14) and (16) into Eq. (5) and then equating each coefficient of the same order power of $\phi^{i}(i=0,1,2, \cdots, 9)$ to zero yields a system of nonlinear equations for $a_{0}, a_{1}$, $a_{2}, \quad \cdots, a_{m}, k$ and $\omega$ :

$$
\begin{aligned}
& \phi^{0}:-\frac{224}{3} a_{0}^{3} a_{1} k \sigma+a_{1} \omega \sigma+112 a_{0}^{2} a_{1} k^{3} \sigma^{2}+70 a_{1}^{3} k^{3} \sigma^{3}+504 a_{0} a_{1} a_{2} k^{3} \sigma^{3}-224 a_{0} a_{1} k^{5} \sigma^{3} \\
& -1120 a_{1} a_{2} k^{5} \sigma^{4}+272 a_{1} k^{7} \sigma^{4}=0, \\
& \phi^{1}:-224 a_{0}^{2} a_{1}^{2} k \sigma-\frac{448}{3} a_{0}^{3} a_{2} k \sigma+2 a_{2} \omega \sigma+728 a_{0} a_{1}^{2} k^{3} \sigma^{2}+896 a_{0}^{2} a_{2} k^{3} \sigma^{2} \\
& +924 a_{1}^{2} a_{2} k^{3} \sigma^{3}+1008 a_{0} a_{2}^{2} k^{3} \sigma^{3}-1344 a_{1}^{2} k^{5} \sigma^{3}-3808 a_{0} a_{2} k^{5} \sigma^{3}-4256 a_{2}^{2} k^{5} \sigma^{4} \\
& +7936 a_{2} k^{7} \sigma^{4}=0 \text {, } \\
& \phi^{2}:-\frac{224}{3} a_{0}^{3} a_{1} k+a_{1} \omega-224 a_{0} a_{1}^{3} k \sigma-672 a_{0}^{2} a_{1} a_{2} k \sigma+448 a_{0}^{2} a_{1} k^{3} \sigma+826 a_{1}^{3} k^{3} \sigma^{2} \\
& +5544 a_{0} a_{1} a_{2} k^{3} \sigma^{2}-1904 a_{0} a_{1} k^{5} \sigma^{2}+2352 a_{1} a_{2}^{2} k^{3} \sigma^{3}-18424 a_{1} a_{2} k^{5} \sigma^{3} \\
& +3968 a_{1} k^{7} \sigma^{3}=0 \text {, } \\
& \phi^{3}:-224 a_{0}^{2} a_{1}^{2} k-\frac{448}{3} a_{0}^{3} a_{2} k+2 a_{2} \omega-\frac{224}{3} a_{1}^{4} k \sigma-896 a_{0} a_{1}^{2} a_{2} k \sigma-448 a_{0}^{2} a_{2}^{2} k \sigma \\
& +1904 a_{0} a_{1}^{2} k^{3} \sigma+2240 a_{0}^{2} a_{2} k^{3} \sigma+6412 a_{1}^{2} a_{2} k^{3} \sigma^{2}+6832 a_{0} a_{2}^{2} k^{3} \sigma^{2}-6328 a_{1}^{2} k^{5} \sigma^{2} \\
& -17248 a_{0} a_{2} k^{5} \sigma^{2}+1568 a_{2}^{3} k^{3} \sigma^{3}-36176 a_{2}^{2} k^{5} \sigma^{3}+56320 a_{2} k^{7} \sigma^{3}=0 \text {, } \\
& \phi^{4}:-224 a_{0} a_{1}^{3} k-672 a_{0}^{2} a_{1} a_{2} k+336 a_{0}^{2} a_{1} k^{3}-\frac{1120}{3} a_{1}^{3} a_{2} k \sigma-1120 a_{0} a_{1} a_{2}^{2} k \sigma \\
& +1666 a_{1}^{3} k^{3} \sigma+10920 a_{0} a_{1} a_{2} k^{3} \sigma-3360 a_{0} a_{1} k^{5} \sigma+12992 a_{1} a_{2}^{2} k^{3} \sigma^{2} \\
& -59864 a_{1} a_{2} k^{5} \sigma^{2}+12096 a_{1} k^{7} \sigma^{2}=0, \\
& \phi^{5}:-\frac{224}{3} a_{1}^{4} k-896 a_{0} a_{1}^{2} a_{2} k-448 a_{0}^{2} a_{2}^{2} k+1176 a_{0} a_{1}^{2} k^{3}+1344 a_{0}^{2} a_{2} k^{3}-672 a_{1}^{2} a_{2}^{2} k \sigma \\
& -448 a_{0} a_{2}^{3} k \sigma+10948 a_{1}^{2} a_{2} k^{3} \sigma+11536 a_{0} a_{2}^{2} k^{3} \sigma-8848 a_{1}^{2} k^{5} \sigma-23520 a_{0} a_{2} k^{5} \sigma \\
& +7616 a_{2}^{3} k^{3} \sigma^{2}-93072 a_{2}^{2} k^{5} \sigma^{2}+129024 a_{2} k^{7} \sigma^{2}=0, \\
& \phi^{6}:-\frac{1120}{3} a_{1}^{3} a_{2} k-1120 a_{0} a_{1} a_{2}^{2} k+910 a_{1}^{3} k^{3}+5880 a_{0} a_{1} a_{2} k^{3}-1680 a_{0} a_{1} k^{5} \\
& -\frac{1568}{3} a_{1} a_{2}^{3} k \sigma+20048 a_{1} a_{2}^{2} k^{3} \sigma-69608 a_{1} a_{2} k^{5} \sigma+13440 a_{1} k^{7} \sigma=0, \\
& \phi^{7}:-672 a_{1}^{2} a_{2}^{2} k-448 a_{0} a_{2}^{3} k+5460 a_{1}^{2} a_{2} k^{3}+5712 a_{0} a_{2}^{2} k^{3}-3864 a_{1}^{2} k^{5}-10080 a_{0} a_{2} k^{5} \\
& -\frac{448}{3} a_{2}^{4} k \sigma+10976 a_{2}^{3} k^{3} \sigma-95088 a_{2}^{2} k^{5} \sigma+120960 a_{2} k^{7} \sigma=0, \\
& \phi^{8}:-\frac{1568}{3} a_{1} a_{2}^{3} k+9408 a_{1} a_{2}^{2} k^{3}-27048 a_{1} a_{2} k^{5}+5040 a_{1} k^{7}=0, \\
& \phi^{9}:-\frac{448}{3} a_{2}^{4} k+4928 a_{2}^{3} k^{3}-33936 a_{2}^{2} k^{5}+40320 a_{2} k^{7}=0 \text {. }
\end{aligned}
$$

With the help of Mathematica 4.0, solving the system of Eqs. (17)-(26) we have $a_{0}=k^{2} \sigma, \quad a_{1}=0, \quad a_{2}=\frac{3}{2} k^{2}, \quad \omega=-\frac{4}{3} k^{7} \sigma^{3}$, 
and hence obtain

$$
u=k^{2} \sigma+\frac{3}{2} k^{2} \phi^{2}(\xi), \quad \xi=k x-\frac{4}{3} k^{7} \sigma^{3} t .
$$

Since the subsidiary equation $\phi^{\prime}=\sigma+\phi^{2}$ has solutions [18]:

$$
\phi(\xi)=\left\{\begin{array}{l}
-\sqrt{-\sigma} \tanh (-\sqrt{-\sigma} \xi), \quad \sigma<0, \\
-\sqrt{-\sigma} \operatorname{coth}(-\sqrt{-\sigma} \xi), \quad \sigma<0, \\
\sqrt{\sigma} \tan (\sqrt{\sigma} \xi), \quad \sigma>0, \\
-\sqrt{\sigma} \cot (\sqrt{\sigma} \xi), \quad \sigma>0, \\
-\frac{1}{\xi}, \quad \sigma=0 .
\end{array}\right.
$$

We then obtain hyperbolic function solutions

$$
\begin{aligned}
& u=k^{2} \sigma-\frac{3}{2} k^{2} \sigma \tanh ^{2}(-\sqrt{-\sigma} \xi), \quad \xi=k x-\frac{4}{3} k^{7} \sigma^{3} t, \\
& u=k^{2} \sigma-\frac{3}{2} k^{2} \sigma \operatorname{coth}^{2}(-\sqrt{-\sigma} \xi), \quad \xi=k x-\frac{4}{3} k^{7} \sigma^{3} t,
\end{aligned}
$$

Trigonometric function solutions

$$
\begin{aligned}
& u=k^{2} \sigma+\frac{3}{2} k^{2} \sigma \tan ^{2}(\sqrt{\sigma} \xi), \quad \xi=k x-\frac{4}{3} k^{7} \sigma^{3} t, \\
& u=k^{2} \sigma-\frac{3}{2} k^{2} \sigma \operatorname{coth}^{2}(-\sqrt{-\sigma} \xi), \quad \xi=k x-\frac{4}{3} k^{7} \sigma^{3} t,
\end{aligned}
$$

and rational solution of Eq. (3):

$$
u=\frac{3 k^{2}}{2 \xi^{2}}, \quad \xi=k x-\frac{4}{3} k^{7} \sigma^{3} t .
$$

\section{Conclusion}

Since Eqs. (17)-(26) exist a set of solutions listed in Eq. (27), the mathematical model (3) has been verified to pass through the test of solvability on computer by using Mathematica 4.0 software system combined with a subsidiary equation method. Based on the Eq. (28), five solutions including hyperbolic function solutions, trigonometric function solutions and rational solution are obtained. It is shown that the Mathematica 4.0 software system combined with the subsidiary equation method can provide a preferred mathematical tool for testing the solvability of some new nonlinear differential equations.

\section{Acknowledgement}

This work was supported by the Natural Science Foundation of China (11547005), the PhD Start-up Fund of Liaoning Province of China (20141137), the Liaoning BaiQianWan Talents Program (2013921055) and the Natural Science Foundation of Liaoning Province of China (L2012404).

\section{References}

[1] Xiaoying Shan, Junyi Zhu. The Mikhauilov-Novikov-Wang hierarchy and its Hamiltonian structures. Acta Physica Polonica B, 43 (2012) 1953-1963.

[2] Clifford Garder, John Greene, Martin Kruskal, Robert Miura. Method for solving the Korteweg-de Vries equation. Physical Review Letters, 19 (1967) 1095-1097.

[3] Robert Miura. Bäcklund Transformation. Springer: Berlin, Germany, 1978. 
[4] Ryogo Hirota. Exact solution of the Korteweg-de Vries equation for multiple collisions of solitons. Physical Review Letters, 27 (1971) 1192-1194.

[5] Mingliang Wang. Exact solutions for a compound KdV-Burgers equation. Physics Letters A, 213 (1996) 279-287.

[6] Sheng Zhang, Tiecheng Xia. Symbolic computation and new families of exact non-travelling wave solutions of (3+1)-dimensional Kadomstev-Petviashvili equation. Applied Mathematics and Computation, 181 (2006) 319-331.

[7] Shikuo Liu, Zuntao Fu, Shida Liu, Qiang Zhao. Jacobi elliptic function expansion method and periodic wave solutions of nonlinear wave equations. Physics Letters A, 289 (2001) 69-74.

[8] Sheng Zhang, Meitong Chen, Weiyi Qian. Painleve analysis for a forced Korteveg-de Vries equation arisen in fluid dynamics of internal solitary waves, Thermal Science, 19 (2015) 1223-1226.

[9] Sheng Zhang, Tiecheng Xia. A generalized auxiliary equation method and its application to (2+1)-dimensional asymmetric Nizhnik-Novikov-Vesselov equations, Journal of Physics A: Mathematical and Theoretical, 40 (2006) 227-248

[10] Sheng Zhang, Xudong Gao. Exact N-soliton solutions and dynamics of a new AKNS equations with time-dependent coefficients, Nonlinear Dynamics, 83(2016) 1043-1052.

[11] Engui Fan. Travelling wave solutions in terms of special functions for nonlinear coupled evolution systems. Physics Letters A, 300 (2002) 243-249.

[12] Sheng Zhang, Hongqing Zhang. A transformed rational function method for (3+1)-dimensional potential YTSF equation. Pramana-Journal of Physics, 76 (2011) 561-571.

[13] Sheng Zhang, Bin Cai, Bo Xu. Variable separation method for nonlinear time fractional biological population model. International Journal of Numerical Methods for Heat and Fluid Flow, 25 (2015) 1531-1541.

[14] Sheng Zhang, Dongdong Liu. The third kind of Darboux transformation and multisoliton solutions for generalized Broer-Kaup equations. Turkish Journal of Physics, 39 (2015) 165-177.

[15] Sheng Zhang, Tiecheng Xia. Variable-coefficient Jacobi elliptic function expansion method for (2+1)-dimensional Nizhnik-Novikov-Vesselov equations. Applied Mathematics and Computation, 218 (2011) 1308-1316.

[16] Sheng Zhang, Hongqing Zhang. Fan sub-equation method for Wick-type stochastic partial differential equations. Physics Letters A, 374 (2010) 4180-4187.

[17] Sheng Zhang, Ling Dong, Jin-Mei Ba, Ying-Na Sun. The (G'/G)-expansion method for discrete nonlinear Schrödinger equation. Pramana-Journal of Physics, 74 (2010) 207-218.

[18] Sheng Zhang, Hongqing Zhang. Fractional sub-equation method and its applications to nonlinear fractional PDEs. Physics Letters A, 375 (2011) 1069-1073. 